\title{
UNA NUEVA ESPECIE DE ARISTOLOCHIA (ARISTOLOCHIACEAE) DE CHIAPAS, MEXICO
}

\author{
Jose F. Ortega Ortiz \\ Y \\ Roberto V. Ortega Ortiz \\ Herbario "Arturo Gómez-Pompa" XALU \\ Facultad de Biología, Xalapa \\ Universidad Veracruzana \\ Apartado postal 785, Zona Universitaria \\ Xalapa, Veracruz
}

\section{RESUMEN}

Se describe Aristolochia chiapensis, una liana que habita la vegetación secundaria derivada de selva alta perennifolia. Esta planta crece a orillas de ríos o lagos de la parte nororiental del estado de Chiapas, principalmente en la región de la Selva Lacandona. El nuevo taxon pertenece a un grupo de especies estrechamente relacionadas (Aristolochia asclepiadifolia Brandg., A. paracleta Pfeifer, A. steyermarkii Standl., A. veracruzana Ortega), con las que comparte la presencia de tres lóbulos estigmáticos y seis estambres unidos en un ginostemo. Sin embargo, se diferencia de los demás componentes de este conjunto por la inflorescencia, el cáliz y lóbulo central del limbo de mayor tamaño, así como por la coloración blanquecina y la superficie rugosa en el interior del utrículo del cáliz.

\begin{abstract}
Aristolochia chiapensis, a woody vine inhabiting secondary vegetation derived from high evergreen forest, is described. This species grows in margins of rivers and lakes in the northeastern portion of Chiapas, Mexico, mainly in the Lacandon rainforest. This new taxon belongs to a group of closely related species (Aristolochia asclepiadifolia Brandg., A. paracleta Pfeifer, A. steyermarkii Standl., A. veracruzana Ortega), sharing the presence of three stigmatic lobes and six stamens united into a gynostemium. However, it differs from the remaining members of this group in its bigger inflorescence, calyx and medium lobe of the limb, as well as in the whitish coloration and rugose surface inside the calyx utricle.
\end{abstract}

Chiapas ha sido considerado como uno de los tres estados del país con mayor diversidad vegetal, con más de 8248 especies (Breedlove, 1986). Sin embargo, como producto de colectas intensivas en los últimos años, esta cifra ha aumentado con aproximadamente 1000 registros adicionales y varias novedades para la ciencia (Toledo, 1994).

Pfeifer en sus trabajos sobre Aristolochia (1966, 1970a y 1970b) cita para México 55 especies, 8 de ellas de Chiapas (2 de la sección Pentandrae y 6 de la sección 
Hexandrae). Esta cifra ha aumentado a 16 con la reciente información proveniente de la bibliografía y de ejemplares de herbario.

Como parte de una serie de trabajos que se pretenden realizar sobre la familia Aristolochiaceae en México, se detectó la existencia de una especie hexandra desconocida del género, que se describe a continuación.

Aristolochia chiapensis J. Ortega \& R. Ortega, sp. nov. Fig. 1.

Frutex volubilis. Folia elliptica vel lanceolata. Inflorescentia $15-21.8 \mathrm{~cm}$ longa; pedunculus $4.5-6 \mathrm{~cm}$ longus. Calyx purpureus, $9-11 \mathrm{~cm}$ longus, $10-12 \mathrm{~cm}$ latus, utriculus albus, rugosus, puberulus omnino superficiei interna, in basem purpureus, limbus 3-lobatus, lobus medius lateralibus major, $5-10 \mathrm{~cm}$ longus, $6-12 \mathrm{~cm}$ latus, purpureus, inflatus, columna 3-lobata, stipitata, 4-8 mm alta. Fructus capsularis, ferrugineus, $6-8 \mathrm{~cm}$ longus, $1.5-2 \mathrm{~cm}$ diametro, dense puberulus.

Bejuco voluble, de 2 a $8 \mathrm{~m}$ de largo, tallo cilíndrico, brillante, densamente velutino en la juventud, glabrescente al madurar; corteza suberosa y glabrescente. Hojas de color verde oscuro, elípticas, a veces lanceoladas, $10-20 \mathrm{~cm}$ de largo, $4.5-11 \mathrm{~cm}$ de ancho, membranáceas o semicoriáceas, haz opaco y pubescente en hojas jóvenes, opaco y glabrescente en las maduras, envés densamente pubescente o viloso, epinervio, margen entero, ápice agudo o algunas veces apiculado, base redondeada o ligeramente oblicua; nervación reticulada, con una costa, 7-9 pares de nervios primarios, actinódromos; pecíolo cilíndrico, 1-1.5 cm de largo, 1-2 mm de diámetro, densamente pubescente, canescente; seudoestípulas ausentes. Inflorescencia solitaria, axilar, racemosa, 15-21.8 cm de largo; pedúnculo cilíndrico, 4.5-6 cm de largo, 0.5-1.2 mm de diámetro, opaco, densamente pubérulo o pubescente, ferrugíneo, bracteolas lanceoladas, sésiles, 1-3 mm de largo, ca. $1 \mathrm{~mm}$ de ancho, foliáceas, densamente pubescentes, ferrugíneas, margen entero, ápice agudo, base abrazadora; pedicelo cilíndrico, $1.5-2 \mathrm{~cm}$ de largo, $0.5-1 \mathrm{~mm}$ de diámetro, opaco, densamente pubérulo o pubescente. Cáliz geniculado, de color púrpura, $9-13.8 \mathrm{~cm}$ de largo, 10-12 cm de ancho, carnoso, brillante, puberulento en la superficie externa; utrículo contraído hacia la base, $2-3.5 \mathrm{~cm}$ de largo, $1-1.5 \mathrm{~cm}$ de ancho, de color blanco, brillante con rugosidades y pubérulo en toda la superficie interna, con una franja morada hacia la base, siringe urceolada, blanca y extendida hacia el interior del utrículo, 1-1.5 mm de largo, $7 \mathrm{~mm}$ de diámetro, brillante y glabra, tubo no definido por la constricción del utrículo con el limbo; limbo 3 lobado, purpúreo o rojo, los lóbulos desiguales, el central ovado, delgado e inflado, de mayor tamaño que los laterales, 5-10 cm de largo, 6-12 cm de ancho, brillantes, pubérulos en la superficie interna, el margen entero y extendido, el ápice agudo o algunas veces con un pequeño apéndice de $2-3 \mathrm{~mm}$ de largo; estambres 6 , unidos en un ginostemo estipitado; anteras sésiles, 2 por lóbulo, con dehiscencia longitudinal, amarillas, elipsoides, 3-5 mm de largo, 0.5-1 mm de ancho, glabras; ovario ínfero, tricarpelar, 6 locular, de color moreno claro, cilíndrico, 1.5-2 cm de largo, 2-3 mm de diámetro, opaco, densamente pubescente o pubérulo, ferrugíneo; placentación axilar, los óvulos numerosos; ginostemo columnar, 4-8 mm de largo, 3-4 mm de diámetro, carnoso, opaco y glabro, con 3 lóbulos estigmáticos, triangulares, 2-2.5 mm de largo, 2-3 mm de ancho, brillantes y glabros, ápice agudo, margen revoluto. Fruto capsular, de color moreno claro, claviforme en estado joven 

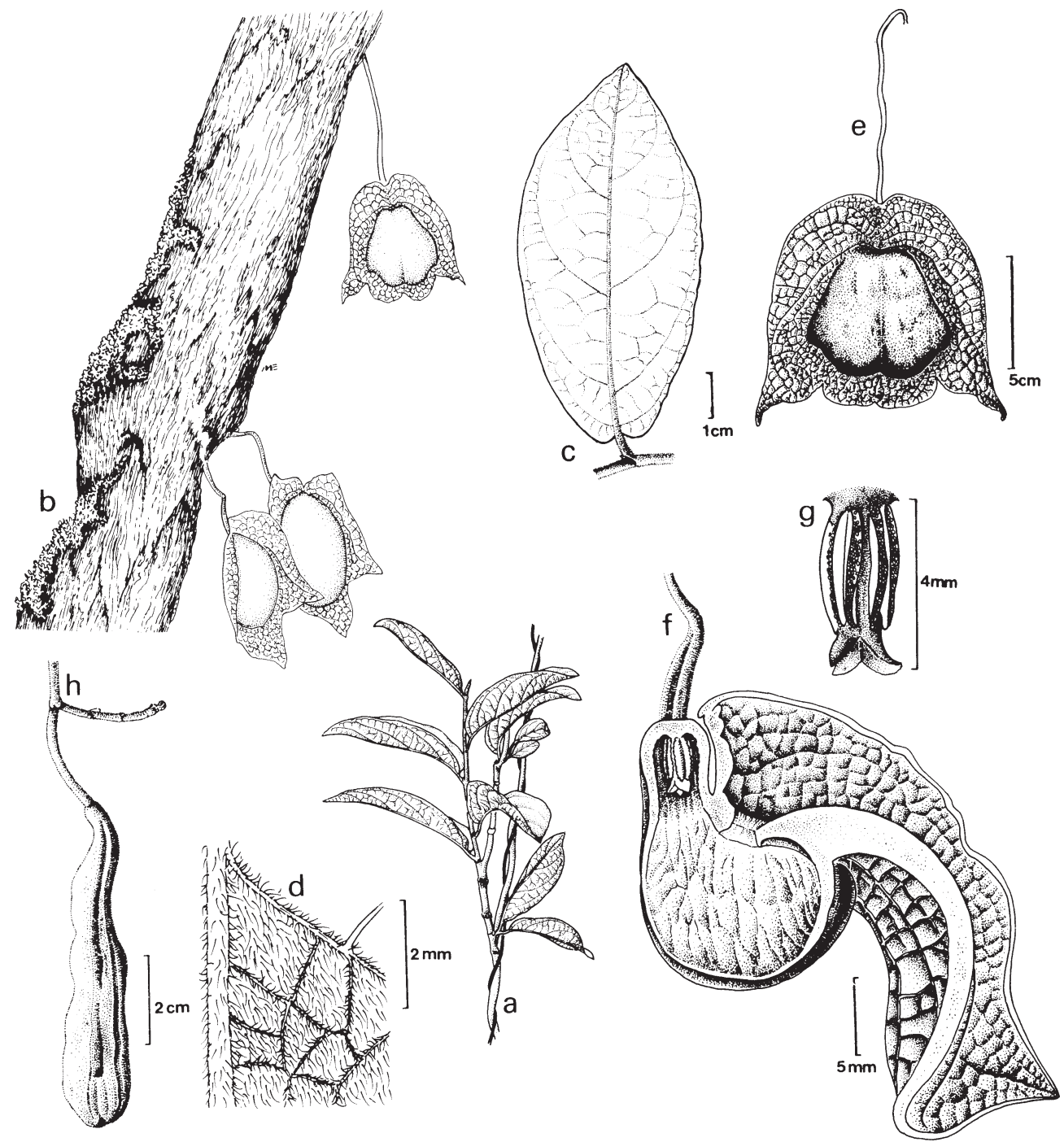

Fig. 1. Aristolochia chiapensis. a. hábito; b. tallo maduro con flores; c. hoja; d. detalle del envés de la hoja; e. flor; f. corte longitudinal mostrando las partes estructurales de la flor; g. detalle del ginostemo; h. fruto. Ilustración de M. Escamilla, basada en fotografías y en los ejemplares J. Ortega 0. 528 y 537. 
e inmaduro, ca. 6-8 cm de largo, $1.5-2 \mathrm{~cm}$ de diámetro, 6 valvado, opaco, densamente pubérulo y ferrugíneo, ápice truncado, base curvada, de dehiscencia septífraga; semillas numerosas, triangulares y pequeñas.

TIPO: México, Chiapas, mpio. Palenque, $3-5$ km de Palenque, junto al Río Chacamax, $17^{\circ} 30^{\prime} \mathrm{N}, 91^{\circ} 57^{\prime} \mathrm{O}$, suelo arenoso en acahuales de selva alta perennifolia cercanos al río, alt. 100 m, 06.II.1987 (fl. y fr.), J. Ortega O. 528 (Holotipo: XAL; isotipos: CHAPA, ENCB, GH, MEXU, NY, TEX, US, XALU).

Material adicional revisado: México, Chiapas, mpio. Ocosingo, a $4 \mathrm{~km}$ al $\mathrm{W}$ de Crucero Corozal, camino Palenque-Boca Lacantum, alt. 180 m, 19.IX.1984 (fl.), E. Martínez S. 7595 (MEXU, XAL); mpio. Ocosingo, Río Cedro al W de Lacanjá Chanzayab, alt. 300 m, 21.VII.1987 (fl.), J. Ortega e I. Calzada 537(XAL); mpio. Ocosingo, Río Cedro al W de Lacanjá Chanzayab, alt. 300 m, 15.I.1984 (fr.) F. Vázquez B. y S. Avendaño 1506-b (XAL); mpio. Palenque, Palenque, junto al Río Chacamax, 22.I.1955, S. Fuentes s.n. (XAL); mpio. Palenque, Palenque, junto al Río Chacamax, alt. 100 m, 22.VII.1987 (fl.), J. Ortega e I. Calzada 540 (XAL).

Aristolochia chiapensis sólo se conoce del NE de Chiapas, se encuentra en acahuales de selva alta perennifolia, donde crece en sitios cercanos a los ríos o en lugares muy húmedos, en suelos arcillo-arenosos con abundante humus, entre los 100 y 350 m s.n.m.

A. chiapensis se conoce localmente como "itamo real" y se le atribuyen en la región de Palenque propiedades medicinales (principalmente a la raíz, la cual es utilizada como alexítera).

Este taxon pertenece a un complejo de especies muy relacionadas formado por $A$. veracruzana Ortega, A. asclepiadifolia Brandg., A. paracleta Pfeifer y A. steyermarkii Standl., con las que comparte la presencia de tres lóbulos estigmáticos y seis estambres unidos en un ginostemo. Sin embargo, como puede apreciarse en el Cuadro 1, las diferencias que la separan claramente de las demás especies justifican su reconocimiento.

\section{AGRADECIMIENTOS}

Agradecemos a los directores y encargados de los herbarios MEXU y XAL, quienes amablemente pusieron a nuestra disposición los ejemplares que se requirieron para el presente trabajo, y a la bióloga Carlota de Léon Aguirre, directora de la Facultad de Biología de la Universidad Veracruzana, por proporcionarnos su apoyo en la publicación del presente artículo. Asimismo, hacemos patente el agradecimiento al técnico Juan Ismael Calzada por la ayuda prestada para la colecta del material botánico, y al biólogo Jorge Benítez Rodríguez, por la traducción del resumen al inglés. 
Ortega y Ortega: Una Nueva Especie de Aristolochia de Chiapas, México

Cuadro 1. Características diferenciales comparativas entre Aristolochia chiapensis y especies afines.

\begin{tabular}{|c|c|c|c|c|c|}
\hline & A. chiapensis & A. veracruzana & A. paracleta & A. asclepiadifolia & A. steyermarkii \\
\hline \multicolumn{6}{|l|}{ Inflorescencia } \\
\hline posición & $\begin{array}{l}\text { axilar y/o } \\
\text { cauliflora }\end{array}$ & $\begin{array}{l}\text { axilar y/o } \\
\text { cauliflora }\end{array}$ & axilar & $\begin{array}{l}\text { axilar y/o } \\
\text { cauliflora }\end{array}$ & axilar \\
\hline tamaño & $\begin{array}{c}15-21.8 \mathrm{~cm} \\
\text { de largo }\end{array}$ & $\begin{array}{l}8-11 \mathrm{~cm} \\
\text { de largo }\end{array}$ & $\begin{array}{l}3-6 \mathrm{~cm} \\
\text { de largo }\end{array}$ & $\begin{array}{l}5-8 \mathrm{~cm} \\
\text { de largo }\end{array}$ & $\begin{array}{l}\text { 6-7.5 cm } \\
\text { de largo }\end{array}$ \\
\hline \multicolumn{6}{|l|}{ Cáliz } \\
\hline \multirow[t]{2}{*}{ tamaño } & $\begin{array}{l}9-13.8 \mathrm{~cm} \\
\text { de largo }\end{array}$ & $\begin{array}{l}4-8 \mathrm{~cm} \\
\text { de largo }\end{array}$ & $\begin{array}{l}4-6 \mathrm{~cm} \\
\text { de largo }\end{array}$ & $\begin{array}{l}1.5-5.3 \mathrm{~cm} \\
\text { de largo }\end{array}$ & $\begin{array}{l}3-5.5 \mathrm{~cm} \\
\text { de largo }\end{array}$ \\
\hline & $\begin{array}{l}10-12 \mathrm{~cm} \\
\text { de ancho }\end{array}$ & $\begin{array}{c}3-5 \mathrm{~cm} \\
\text { de ancho }\end{array}$ & $\begin{array}{c}2-3 \mathrm{~cm} \\
\text { de ancho }\end{array}$ & $\begin{array}{l}1.5-5 \mathrm{~cm} \\
\text { de ancho }\end{array}$ & $\begin{array}{c}4-5 \mathrm{~cm} \\
\text { de ancho }\end{array}$ \\
\hline \multicolumn{6}{|l|}{ Utrículo } \\
\hline color & blanco & moreno oscuro & purpúreo & amarillo & purpúreo \\
\hline $\begin{array}{l}\text { indumento } \\
\text { interno }\end{array}$ & $\begin{array}{l}\text { rugoso y } \\
\text { pubérulo }\end{array}$ & $\begin{array}{l}\text { rugoso y } \\
\text { pubérulo }\end{array}$ & áspero, glabro & liso y glabro & $\begin{array}{l}\text { rugoso y } \\
\text { pubérulo }\end{array}$ \\
\hline \multicolumn{6}{|l|}{$\begin{array}{l}\text { Lóbulo central } \\
\text { del limbo }\end{array}$} \\
\hline tamaño & $\begin{array}{l}\text { 6-12 cm } \\
\text { de ancho }\end{array}$ & $\begin{array}{l}3.5-5 \mathrm{~cm} \\
\text { de ancho }\end{array}$ & $\begin{array}{c}2-3 \mathrm{~cm} \\
\text { de ancho }\end{array}$ & $\begin{array}{l}3-4.5 \mathrm{~cm} \\
\text { de ancho }\end{array}$ & $\begin{array}{l}2.3-4.5 \mathrm{~cm} \\
\text { de ancho }\end{array}$ \\
\hline consistencia & $\begin{array}{l}\text { delgado e } \\
\text { inflado }\end{array}$ & $\begin{array}{c}\text { poco carnoso } \\
\text { e inflado }\end{array}$ & $\begin{array}{c}\text { muy carnoso } \\
\text { e inflado }\end{array}$ & $\begin{array}{l}\text { carnoso e } \\
\text { inflado }\end{array}$ & $\begin{array}{l}\text { carnoso e } \\
\text { inflado }\end{array}$ \\
\hline \multicolumn{6}{|l|}{ Fruto } \\
\hline tamaño & $\begin{array}{l}6-8 \mathrm{~cm} \\
\text { de largo }\end{array}$ & $\begin{array}{l}4.5-5 \mathrm{~cm} \\
\text { de largo }\end{array}$ & $\begin{array}{l}\text { 8-10 cm } \\
\text { de largo }\end{array}$ & $\begin{array}{l}5-7.5 \mathrm{~cm} \\
\text { de largo }\end{array}$ & $\begin{array}{l}5 \mathrm{~cm} \text { de } \\
\text { largo }\end{array}$ \\
\hline color & café claro & verde claro & café claro & verde claro & café oscuro \\
\hline indumento & pubérulo & $\begin{array}{l}\text { piloso o } \\
\text { pubescente }\end{array}$ & $\begin{array}{l}\text { pubérulo o } \\
\text { glabro }\end{array}$ & $\begin{array}{c}\text { pubescente o } \\
\text { pubérulo }\end{array}$ & $\begin{array}{l}\text { pubérulo y } \\
\text { glabrescente }\end{array}$ \\
\hline
\end{tabular}




\section{LITERATURA CITADA}

Breedlove, D. E. 1986. Listados florísticos de México. IV. Flora de Chiapas. Instituto de Biología, Universidad Nacional Autónoma de México. México, D.F. 246 pp.

Pfeifer, H. W. 1966. Revision of the North and Central American hexandrous species of Aristolochia (Aristolochiaceae). Ann. Missouri Bot. Gard. 53: 115-196.

Pfeifer, H. W. 1970a. Two new hexandrous Mexican Aristolochias. The University of Connecticut Occasional Papers, Biological Science Series 1(4): 291-297.

Pfeifer, H. W. 1970b. A taxonomic revision of the pentandrous species of Aristolochia. The University of Connecticut Publication Series. Storrs. $134 \mathrm{pp}$.

Toledo, V. M. 1994. La diversidad biológica de México. Nuevos retos para la investigación en los noventas. Ciencias 34: 43-59.

Aceptado para publicación en octubre de 1997. 